PESQUIMAT Revista de la Fac.CC.MM.de la

Universidad Nacional Mayor de San Marcos

Vol.III, $N^{\circ} 2$, Julio 2000, pag.1-13. Lima-Perú

\title{
SET-THEORETIC COMPLETE INTERSECTIONS ON BINOMIALS, THE SIMPLICIAL TORIC CASE
}

\author{
Margherita Barile ${ }^{1}$, Marcel Morales ${ }^{2}$, Apostolos Thoma ${ }^{3}$
}

\begin{abstract}
Let $V$ be a simplicial toric variety of codimension $r$ over a field of any characteristic. We completely characterize the implicial toric varieties that are set-theoretic complete intersections on binomials. In particular we prove that:

1. In characteristic zero, $V$ is a set-theoretic complete intersection on binomials if and only if $V$ is a complete intersection. Moreover, if $F_{1}, \ldots, F_{r}$ are binomials such that $I(V)=\operatorname{rad}\left(F_{1}, \ldots, F_{r}\right)$, then $I(V)=\left(F_{1}, \ldots, F_{r}\right)$. We also get a geometric proof of some of the results in [9] characterizing complete intersections by gluing semigroups.

2. In positive characteristic $p, V$ is a set-theoretic complete intersection on binomials if and only if $V$ is completely p-glued.

These results improve and complete all known results on these topics.
\end{abstract}

\section{INTRODUCTION}

The determination of the minimum number of equations needed to define an algebraic variety $V$ set-theoretically or ideal-theoretically is an old and important problem in Algebraic Geometry. In this paper we consider the case of simplicial toric varieties and it turns out that these two problems are strongly related in characteristic zero, see Theorem 1.

The ideal of a toric variety is a prime binomial ideal. A binomial ideal is an ideal generated by binomials. Eisenbud and Sturmfels began the systematic study of binomial ideals in [4], where also the ubiquity of binomial ideals was presented. There are numerous publications in

\footnotetext{
${ }^{1}$ Dipartimento di Matematica,Università degli Studi di Bari,Via Orabona 4, 70125 Bari (Italy)

${ }^{2}$ Université de Grenoble I, Institut Fourier, URA 188, B.P.74, 38402 Saint-Martin D'Hères Cedex, and IUFM de Lyon, 5 rue Anselme,69317 Lyon Cedex (France)

${ }^{3}$ Department of Mathematics, University of Ioannina, Ioannina 45110 (Greece)
} 
recent years on binomial ideals, and several of them treat the problem of the 20 minimal generation of a binomial ideal or of the radical of it, for example: $[1,2,5,6,7,9,10,12]$.

The binomial arithmetical rank of a binomial ideal $I$ (written bar $(I)$ ) is the smallest integer $s$ for which there exist binomials $f_{1}, \ldots, f_{s}$ in $I$ such that $\operatorname{rad}(I)=\operatorname{rad}\left(f_{1}, \ldots, f_{s}\right)$. Hence the binomial arithmetical rank is an upper bound for the arithmetical rank of a binomial ideal. From the definitions we deduce the following inequality for a binomial ideal $I$ :

$$
h(I) \leq \operatorname{ara}(I) \leq \operatorname{bar}(I) \leq \mu(I) .
$$

Here $h(I)$ denotes the height and $\mu(I)$ denotes the minimal number of generators of $I$. When $h(I)=\operatorname{ara}(I)$ the ideal $I$ is called a settheoretic complete intersection and when $h(I)=\mu(I)$ it is called a complete intersection.

Let $K$ be a field of any characteristic and let $\bar{K}$ be the algebraic closure of $K$. Let $\mathbf{e}_{1}, \ldots, \mathbf{e}_{\mathbf{n}}$ be the elements of the canonical basis of $\mathbb{Z}^{n}$, and for all $i=1, \ldots, r$ let $\mathbf{a}_{\mathbf{1}}=\left(a_{i, 1}, \ldots, a_{i, n}\right) \in \mathbb{N}^{n}$. Let $d_{1} \ldots, d_{n} \in \mathbb{N}$ and set $T=\left\{d_{1} \mathbf{e}_{1}, \ldots, d_{n} \mathbf{e}_{\mathbf{n}}, \mathbf{a}_{\mathbf{1}}, \ldots, \mathbf{a}_{\mathbf{r}}\right\} \subset \mathbb{N}^{n}$. Define

$$
\phi: K\left[x_{1}, \ldots, x_{n}, y_{1}, \ldots, y_{r}\right] \rightarrow K\left[t_{1}, \ldots, t_{n}\right]
$$

as the homomorphism of $K$-algebras for which

$$
\begin{gathered}
\phi\left(x_{i}\right)=\mathbf{t}^{d_{i} \mathbf{e}_{1}} \quad \text { for all } i=1, \ldots, n, \\
\phi\left(y_{i}\right)=\mathbf{t}^{\mathbf{a}_{1}} \quad \text { for all } i=1, \ldots, r .
\end{gathered}
$$

Where $t^{a_{1}}=t_{1}^{a_{i, 1}} \ldots t_{n}^{a_{i, n}}$. Then $\operatorname{Ker} \phi=I_{T}$ is the simplicial toric ideal of $T$ and its affine variety $V=V\left(I_{T}\right)$ of zeros in $K^{n+r}$ is an affine simplicial toric variety in the sense of $[4,11]$, which also includes non normal varieties. The image $\phi\left(K\left[x_{1}, \ldots, x_{n}, y_{1}, \ldots, y_{r}\right]\right)$ is the affine semigroup ring of $T$.

Let $\mathbb{I N T}=\left\{l_{1} d_{1} \mathbf{e}_{1}+\cdots+l_{n+r} \mathbf{a}_{\mathbf{r}}: l_{1}, \ldots, l_{n+r} \in \mathbb{I}\right\}$ be the affine semigroup generated by $T$ and $\mathbb{Z} T=\left\{l_{1} d_{1} \mathbf{e}_{1}+\cdots+l_{n+r} \mathbf{a}_{\mathbf{r}}: l_{1}, \ldots, l_{n+r} \in\right.$ $\mathbb{Z}\}$ the lattice spaned by $T$. We denote the dimension of the lattice by $\operatorname{dim}(\mathscr{Z T})$, which in this case is equal to $n$.

We recall the definition of semigroup gluing.

Definition 1. Let $T_{1}$ and $T_{2}$ be non-empty subsets of $T$ such that $T=$ $T_{1} \cup T_{2}$ and $T_{1} \cap T_{2}=\emptyset$. Then $T$ is called a gluing of $T_{1}$ and $T_{2}$ if there is a nonzero element $\mathbf{a} \in \mathbb{N} T_{1} \cap \mathbb{N} T_{2}$ such that $\mathbb{Z a}=\mathbb{Z} T_{1} \cap \mathbb{Z} T_{2}$. 
The concept of semigroup gluing was defined by J.C. Rosales in [8] and used by K. Fisher, W. Morris and J. Shapiro in [6] to characterize all complete intersections affine semigroups. They proved that for an affine semigroup $\mathbb{I N T}$ which is not a free abelian semigroup it holds: $\mathbb{I N T}$ is a complete intersection if and only if there are two subsets $T_{1}$ and $T_{2}$ of $T$ such that $T$ is the gluing of $T_{1}$ and $T_{2}$ and $\mathbb{I N T} T_{1}, \mathbb{N} T_{2}$ are complete intersection subsemigroups.

Here we also define the notion of $p$-gluing and inductively the notion of completely p-glued, which will be very important for the characterization of set-theoretic complete intersections on binomials in characteristic $p$.

Definition 2. Let $p$ be a prime number and $T_{1}$ and $T_{2}$ be non-empty subsets of $T$ such that $T=T_{1} \cup T_{2}$ and $T_{1} \cap T_{2}=\emptyset$. Then $T$ is called a p-gluing of $T_{1}$ and $T_{2}$ if $\mathbb{Z} T_{1} \cap \mathbb{Z} T_{2}=\mathbb{Z a}^{\prime}$ and there is a nonzero element $\mathrm{a} \in \mathbb{I N T} T_{1} \cap \mathbb{N N T _ { 2 }}$ and there is an integer $k$ such that $\mathbf{a}=p^{k} \mathrm{a}^{\prime}$.

Definition 3. An affine semigroup INT is called completely p-glued if $T$ is the p-gluing of $T_{1}$ and $T_{2}$, where each one of the semigroups

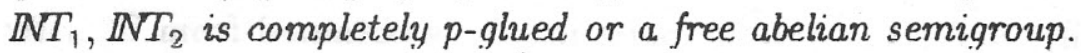

The purpose of this paper is to prove the following results:

1. In characteristic zero the complete intersection simplicial affine toric varieties are characterized as those simplicial affine toric varieties which are set-theoretic complete intersections on binomial hypersurfaces. In this respect one can get a different proof of the characterization of complete intersection simplicial affine semigroups (see [9]) since the characterization of complete intersection affine semigroups is characteristic free. It will be interesting to know if the above property still holds for a general toric variety in characteristic zero.

2. In positive characteristics this result is not true since the class of toric varieties which are set-theoretic complete intersections on binomials is larger than the one of complete intersections. We refer to [1], where we have proved that all simplicial toric varieties with full parametrization are set-theoretic complete intersections on binomials. In positive characteristic $p$, simplicial affine toric varieties which are set-theoretic complete intersections on binomial hypersurfaces are those whose underlying semigroups are com- . pletely $p$-glued.

Throughout the paper we shall refer to the notations introduced in this section. 


\section{MAIN RESULTS}

Let $\Omega=\left\{x_{1}, x_{2}, \ldots, x_{n}, y_{1}, \ldots, y_{r}\right\}$. A partition $\Im$ of $\Omega$ is a set of pairwise disjoint non-empty subsets covering $\Omega$. A partition $\Im_{1}$ refines a partition $\Im_{2}$, denoted by $\Im_{1} \triangleleft \Im_{2}$, if every set of $\Im_{2}$ is the union of some sets of $\Im_{1}$. For all $i=1, \ldots, r$, let $\Pi_{i}=\left(M_{i}, N_{i}\right)$ be an ordered pair of unitary monomials. Let

$$
\Im_{1} \triangleleft \Im_{2} \triangleleft \cdots \triangleleft \Im_{r+1}
$$

be a chain of partitions of $\Omega$. According to a definition given by Delorme [3], the sequence $\Pi_{1}, \ldots, \Pi_{r}$ is called distinguished with respect to this chain if for all $i=1, \ldots, r$ there are $A_{i}, B_{i} \in \Im_{i}$ such that the following two conditions are fulfilled:

- for all $i=1, \ldots, r$,

$$
\operatorname{supp}\left(M_{i}\right) \subset A_{i}, \operatorname{supp}\left(N_{i}\right) \subset B_{i},
$$

- for all $j=2, \ldots, r+1$ the partition $\Im_{j}$ is obtained from $\Im_{j-1}$ by replacing $A_{j}$ and $B_{j}$ by their union.

Definition 4. Let $F \in I=I_{T}$ be a binomial, $F=M-N$, where $M$ and $N$ are unitary monomials. We denote $\Pi(F)=(M, N)$. We also consider the partition $\Im_{1}$ of $\Omega$ formed by the sets

$$
\Im_{1}:\left\{x_{1}, \ldots, x_{n}\right\}, \quad\left\{y_{1}\right\}, \quad \ldots, \quad\left\{y_{r}\right\} .
$$

We shall say that the sequence $\Pi_{1}, \ldots, \Pi_{r}$ of binomial pairs is distinguished if it is distinguished with respect to some chain of partitions starting at $\Im_{1}$.

Let $A \subset \Omega$. We denote by $P_{A}$ the point of $K^{n+r}$ such that its coordinates corresponding to variables belonging to $A$ are equal to 1 and all the others are equal to zero.

Lemma 1. Let $\Im$ be any non trivial partition of $\Omega$ such that $\Im_{1}$ is a refinement of $\Im$, and let $A \in \Im$. Then $P_{A} \notin V=V\left(I_{T}\right)$.

Proof. First suppose that $\left\{x_{1}, \ldots, x_{n}\right\} \subseteq A$. Since $\Im$ is non trivial, we have that $y_{i} \notin A$ for some index $i$. Hence $\bar{y}_{i}=0$, whereas $\bar{x}_{1}=\cdots=$ $x_{n}=1$. Since for any $i$ there exists a binomial $y_{i}^{\alpha_{i}}-M\left(x_{1}, \ldots, x_{n}\right) \in I$, we have that $P_{A} \notin V$. 
Suppose that $\left\{x_{1}, \ldots, x_{n}\right\} \cap A=\emptyset$. Since $\Im_{1}$ is a refinement of $\Im$, we have that $y_{i} \in A$ for some index $i$. Hence $\bar{y}_{i}=1$, whereas $\bar{x}_{1}=\cdots=$ $\bar{x}_{n}=0$. Since for any $i$ there exists a binomial $y_{i}^{\alpha_{i}}-M\left(x_{1}, \ldots, x_{n}\right)$ $\in I$, we have that $P_{A} \notin V$.

Lemma 2. Let $V$ be a simplicial toric variety of codimension $r$ such that bar $(I(V))=r$. Let $F_{1}, \ldots, F_{r}$ be $r$ binomials such that $I(V)=$ $\operatorname{rad}\left(F_{1}, \ldots, F_{r}\right)$. After a suitable re-arrangement of the indices one has that the corresponding sequence $\Pi\left(F_{1}\right), \Pi\left(F_{2}\right), \ldots, \Pi\left(F_{r}\right)$ of binomial pairs is distinguished.

Proof. We are going to give the recursive construction of a chain of $r+1$ partitions starting at $\Im_{1}$ with respect to which the sequence $\Pi\left(F_{1}\right), \ldots, \Pi\left(F_{r}\right)$ is distinguished.

Each of the elements of $\Im_{1}$ contains the support of one of the $2 r$ monomials occurring in $\Pi\left(F_{1}\right), \ldots, \Pi\left(F_{r}\right)$. If this were not true for some $A \in \Im_{1}$, then the point $P_{A}$ would be a point of $V\left(F_{1}, \ldots, F_{r}\right)$, since it would annihilate every monomial in $\Pi\left(F_{1}\right), \ldots, \Pi\left(F_{r}\right)$. But this is a contradiction to Lemma 1.

Since the elements of $\Im_{1}$ are pairwise disjoint, in this way we select $r+1$ different monomials out of the $2 r$ monomials belonging to $\Pi\left(F_{1}\right), \ldots, \Pi\left(F_{r}\right)$. Hence two of the selected monomials, say $M_{1}$ and $N_{1}$, must belong to the same pair. Up to re-arrangements of the indices we may assume that $\Pi\left(F_{1}\right)=\left(M_{1}, N_{1}\right)$. Call $A_{1}$ and $B_{1}$ the elements of $\Im_{1}$ such that $\operatorname{supp}\left(M_{1}\right) \subset A_{1}$ and $\operatorname{supp}\left(N_{1}\right) \subset B_{1}$. Let $\Im_{2}$ be the partition arising from $\Im_{1}$ by replacing the sets $A_{1}$ and $B_{1}$ by their union. We claim that each of the elements of $\Im_{2}$ contains the support of one of the monomials occurring in $\Pi\left(F_{2}\right), \ldots, \Pi\left(F_{r}\right)$. We have to prove the claim only for $A_{1} \cup B_{1}$. Suppose for a contradiction that the claim fails for this set. Then the point $P_{A_{1} \cup B_{1}}$ is a point of $V\left(F_{1}, \ldots, F_{r}\right)$, since $M_{1}\left(P_{A_{1} \cup B_{1}}\right)=1=N_{1}\left(P_{A_{1} \cup B_{1}}\right)$ and each monomial in $\Pi\left(F_{2}\right), \ldots, \Pi\left(F_{r}\right)$ vanishes in $P_{A_{1} \cup R_{1}}$. But this is a contradiction to Lemma 1 . We select $r$ monomials out of the $2(r-1)$ monomials belonging to $\Pi\left(F_{2}\right), \ldots, \Pi\left(F_{r}\right)$, and by the same arguments as in the first part of the proof we conclude that, up to re-arrangements, $\Pi\left(F_{2}\right)=\left(M_{2}, N_{2}\right)$, where $\operatorname{supp}\left(M_{2}\right) \subset A_{2}$ and $\operatorname{supp}\left(N_{2}\right) \subset B_{2}$ for some $A_{2}, B_{2} \in \Im_{2}$. The next steps of the construction are clear: for all $h=2, \ldots, r$ we can construct a partition $\Im_{h+1}$ of $\Omega$ such that:

(a) up to re-arrangements, $\Pi\left(F_{h}\right)=\left(M_{h}, N_{h}\right)$, where $\operatorname{supp}\left(M_{h}\right) \subset A_{h}$ and $\operatorname{supp}\left(N_{h}\right) \subset B_{h}$ for some $A_{h}, B_{h} \in \Im_{h}$;

(b) $\Im_{h+1}$ is obtained from $\Im_{h}$ by replacing $A_{h}$ and $B_{h}$ by their union. These partitions form a chain of the required type. 
Remark 1. For all $i=1, \ldots, r+1$ the partition $\Im_{i}$ consists of $r-i+2$ sets. In particular $\Im_{r}$ consists of 2 sets, namely $A_{r}$ and $B_{r}$. Hence $\Omega=A_{r} \cup B_{r}$. We shall assume that $A_{i} \cap\left\{x_{1}, \ldots, x_{n}\right\}=\emptyset$ for all $1, \ldots, r$. In particular $\left\{x_{1}, \ldots, x_{n}\right\} \subset B_{r}$.

Remark 2. For all $k=1, \ldots, r-1$ we have that $A_{k} \subset A_{r}$ if and only if $B_{k} \subset A_{r}$.

Let us call $z_{i}$, for $i=1, \ldots, g$, the variables in $A_{r}$ and $w_{j}$, for $j=$ $1, \ldots, q$, the variables in $B_{r}$. Note that $g+q=n+r$. Let $\mathbf{b}_{1}$ be the vector of the exponents appearing in the parametrization of $z_{i}$ and $c_{j}$ be the corresponding vector for $w_{j}$. Let $T_{1}=\left\{\mathbf{b}_{1}, \ldots, \mathbf{b}_{\mathbf{g}}\right\}$ and let $T_{2}=\left\{\mathbf{c}_{1}, \ldots, \mathbf{c}_{\mathbf{q}}\right\}$. Then $T=T_{1} \cup T_{2}$ and $T_{1} \cap T_{2}=\emptyset$.

Let $\left\{G_{11}, \ldots, G_{1 s}\right\}$ be the binomials among $F_{1}, \ldots, F_{r-1}$ such that $\operatorname{supp}\left(G_{1 i}\right) \subset A_{r}$, and $\left\{G_{21}, \ldots, G_{2 t}\right\}$ be the binomials such that supp $\left(G_{2 j}\right) \subset B_{r}$. Note that $s+t=r-1$.

Lemma 3. For all $i=2, \ldots, r$ let $s_{i}$ be the number of binomials among $F_{1}, \ldots, F_{i-1}$ whose support is contained in $A_{i}$. Then

$$
\left|A_{i}\right|=s_{i}+1 \text {. }
$$

In particular $\left|A_{r}\right|=s+1$.

Proof. We prove the claim by induction on $i \geq 2$. For $A_{2}$ we have two possible cases. If $\left|A_{2}\right|=1$, then $\operatorname{supp}\left(F_{1}\right) \not \subset A_{2}$, and $s_{2}=0$. If $\operatorname{supp}\left(F_{1}\right) \subset A_{2}$, then $F_{1}=y_{l}^{\alpha}-y_{m}^{\beta}$ for some distinct indices $l, m$ and some positive integers $\alpha, \beta$. But then $A_{1}=\left\{y_{l}\right\}$ and $B_{1}=\left\{y_{m}\right\}$ (or conversely), and $A_{2}=\left\{y_{l}, y_{m}\right\}$. Hence $\left|A_{2}\right|=2$ and $s_{2}=1$.

Now assume that $i>2$ and suppose the claim proven for all $A_{k}$ such that $k<i$. Note that the claim is also true for all $B_{k}$ such that $k<i$ and $\left\{x_{1}, \ldots, x_{n}\right\} \cap B_{k}=\emptyset$. If $\left|A_{i}\right|=1$, then $s_{i}=0$. Suppose that $\left|A_{i}\right|>1$. Then $A_{i}=A_{k} \cup B_{k}$ for some $k<i$. Let $t_{k}$ be the number of binomials among $F_{1} \ldots, F_{k-1}$ whose support is contained in $B_{k}$. Then by induction

$$
\left|A_{k}\right|=s_{k}+1 . \quad \text { and } \quad\left|B_{k}\right|=t_{k}+1
$$

Moreover $s_{k}+t_{k}$ is the number of binomials among $F_{1}, \ldots, F_{k-1}$ whose support is contained in $A_{i}$. We also have that $\operatorname{supp}\left(F_{k}\right) \subset A_{k} \cup B_{k}=A_{i}$. Thus it suffices to show that for all $j=k+1, \ldots, i-1$ it holds: $\operatorname{supp}\left(F_{j}\right) \not \subset A_{i}$. But this is certainly true, because for all $j=k+$ $1 \ldots, i-1$ the set $A_{i}$ is an element of $\Im_{j}$. 
Remark 3. From Lemma 3 we deduce that $g=s+1$ and $q=n+t$.

Remark 4. Note that from the proof of Lemma 3 we deduce that each two vectors in $T_{1}$ are linearly dependent, whence $\operatorname{dim}\left(\mathbb{Z} T_{1}\right)=1$. Note also that $\left\{d_{1} \mathbf{e}_{1}, \ldots d_{n} \mathbf{e}_{\mathbf{n}}\right\} \subset T_{2}$, whence $\operatorname{dim}\left(\mathcal{Z} T_{2}\right)=n$.

Remark 5. Note that $I_{T_{1}}=I_{T} \cap K\left[A_{r}\right]$ and $I_{T_{2}}=I_{T} \cap K\left[B_{r}\right]$.

Lemma 4. We have that $I_{T_{1}}=\operatorname{rad}\left(G_{11}, \ldots G_{1 s}\right)$ and $I_{T_{2}}=\operatorname{rad}\left(G_{21}\right.$, $\left.\ldots, G_{2 t}\right)$.

Proof. We shall prove the Lemma for $I_{T_{1}}$. The binomials $G_{1 i}$ belong to $I_{T_{1}}$, so that $\left(G_{11}, \ldots, G_{1 s}\right) \subset I_{T_{1}}$, whence $V\left(I_{T_{1}}\right) \subset V\left(G_{11}, \ldots, G_{1 s}\right)$. Suppose that the inclusion is strict, then there is a point $\left(y_{1}, \ldots, y_{g}\right)$ of $V\left(G_{11}, \ldots, G_{1 s}\right) \subset \bar{K}^{p}$ which is not a point of $V\left(I_{T_{1}}\right) \subset \bar{K}^{g}$. This means that $\left(y_{1}, \ldots, y_{g}\right)$ cannot be expressed in the form $\left(\mathbf{u}^{\mathbf{b}_{\mathbf{1}}}, \ldots, \mathbf{u}^{\mathbf{b}_{\boldsymbol{z}}}\right)$, for any $\mathrm{u}=\left(u_{1}, \ldots, u_{n}\right) \in \bar{K}^{n}$. Recall that $F_{r}=M_{r}-N_{r}$, where $M_{r}$ is a monomial in the $A_{r}$-variables and $N_{r}$ is a monomial in the $B_{r}$ variables. Let $w=M_{r}\left(y_{1}, \ldots, y_{g}\right)$ and set $N_{r}\left(\mathbf{u}^{\mathbf{c}_{\mathbf{1}}}, \ldots, \mathbf{u}^{\mathbf{c}_{\mathbf{q}}}\right)=\mathbf{u}^{\mathbf{c}}$. Moreover let $\left(v_{1}, \ldots, v_{n}\right) \in \bar{K}^{n}$ be any root of the polynomial $u^{c}-w \in$ $\bar{K}\left[u_{1}, \ldots, u_{n}\right]$. Then the point $\left(y_{1}, \ldots, y_{g} ; \mathbf{v}^{\mathbf{c}_{1}}, \ldots, \mathbf{v}^{\mathbf{c}_{\mathbf{q}}}\right)$ is a point of $V\left(G_{11}, \ldots, G_{1 s}, G_{21}, \ldots, G_{2 t}, F_{r}\right) \subset \bar{K}^{n+r}$ which is not a point of $V$. A contradiction.

\section{ZERO CHARACTERISTIC CASE}

Theorem 1. Let $V$ be a simplicial toric variety of codimension $r$ over a field of characteristic zero. Then bar $(I(V))=r$ if and only if $V$ is a complete intersection. Moreover, if $F_{1}, \ldots, F_{r}$ are binomials such that $I(V)=\operatorname{rad}\left(F_{1}, \ldots, F_{r}\right)$, then $I(V)=\left(F_{1}, \ldots, F_{r}\right)$.

Proof. Suppose that $V$ is a complete intersection, then height $(I(V))=$ $r \leq \operatorname{bar}(I(V)) \leq \mu(I(V))=r$, where $\mu(I(V))$ denotes the minimal number of generators of $I(V)$. Therefore bar $(I(V))=r$.

For the converse suppose that $\operatorname{bar}(I(V))=r$. We proceed by induction on $r \geq 1$. The statement is obvious for $r=1$. Suppose that every simplicial toric variety of codimension smaller than $r$ whose binomial arithmetical rank is equal to the codimension is a complete intersection. We perform the costruction contained in the proof of Lemma 2 for $F_{1}, \ldots, F_{r}$. We suppose that these binomials are arranged in such a way that the corresponding sequence of binomial pairs is distinguished with respect to the chain $\Im_{1} \triangleleft \Im_{2} \triangleleft \cdots \triangleleft \Im_{r+1}$. 
From Lemma 4, Remark 4 and the fact that the Krull dimension of the residue ring of $I_{T_{\mathrm{I}}}$ is equal to the $\operatorname{dim}\left(\mathscr{Z} T_{1}\right)$, see [11], we have $\operatorname{bar}\left(I_{T_{1}}\right)=s=(s+1)-1$, therefore $I_{T_{1}}$ is complete intersection and $I_{T_{1}}=\left(G_{11}, \ldots, G_{1 s}\right)$ by the induction hypothesis. Similarly $\operatorname{bar}\left(I_{T_{2}}\right)=$ $t=(n+t)-n$, therefore $I_{T_{2}}$ is complete intersection and $I_{T_{2}}=$ $\left(G_{21}, \ldots, G_{2 t}\right)$ by the induction hypothesis.

We complete the proof by semigroup gluing. First note that $F_{r}=$ $M_{r}-N_{r} \in I(V)$ and $M_{r}$ only involves variables from $T_{1}$ and $N_{r}$ only variables from $T_{2}$, whence $M_{r}\left(\mathbf{u}^{\mathbf{b}_{\mathbf{1}}}, \ldots, \mathbf{u}^{\mathbf{b}_{\mathbf{z}}}\right)=N_{r}\left(\mathbf{u}^{\mathbf{c}_{\mathbf{1}}}, \ldots, \mathbf{u}^{\mathbf{c}_{\mathbf{q}}}\right)=\mathbf{u}^{\mathrm{a}}$ for some a $\in \mathbb{N} T_{1} \cap \mathbb{N} T_{2}$. We claim that $\mathbb{Z a}=\mathscr{Z} T_{1} \cap \mathbb{Z} T_{2}$, i.e., $T$ is a gluing of $T_{1}$ and $T_{2}$. According to [8] all the preceding assumptions imply that

$$
I(V)=I_{T_{1}}+I_{T_{2}}+\left(F_{r}\right)=\left(G_{11}, \ldots, G_{1 A}, G_{21}, \ldots, G_{2 t}, F_{r}\right)=\left(F_{1}, \ldots, F_{r}\right),
$$

which will complete the proof.

Suppose that our claim is not true. Then there is an element $\mathbf{a}^{\prime} \in \mathbb{Z}^{n}$ such that $\mathbb{Z a}^{\prime}=\mathscr{Z} T_{1} \cap \mathscr{Z} T_{2}$, since $\mathscr{Z} T_{1} \cap \mathscr{Z} T_{2}$ has dimension 1. From a $\in \mathbb{Z} T_{1} \cap \mathbb{Z} T_{2}$ we see that $\mathbf{a} \in \mathbb{Z}^{\prime} \mathbf{a}^{\prime}$, therefore there exists a positive integer $m>1$ such that $\mathbf{a}=m \mathbf{a}^{\prime}$. Note that all entries of a are positive, so that we can assume that the same is true for $\mathbf{a}^{\prime}$.

The degree of an element of $\mathbb{N} N^{n}$ is the sum of its entries, let $d=\operatorname{deg}(\mathbf{a})$ and $d^{\prime}=\operatorname{deg}\left(\mathbf{a}^{\prime}\right)$. Then $d=m d^{\prime}$. Let $\omega \in \bar{K}$ be a primitive $m$-th root of unity and let $\xi \in \bar{K}$ be such that $\xi^{d^{\prime}}=\omega$. Re-arrange the coordinates of $\bar{K}^{n+r}$ in order to form the sequence $\left(z_{1}, \ldots, z_{g} ; w_{1} \ldots, w_{q}\right)$. For all $j=1, \ldots, q$, let $\gamma_{j}=\sum_{k-1}^{n} c_{j, k}$. The point $\left(1, \ldots, 1 ; \xi^{\gamma_{1}}, \ldots, \xi^{\gamma_{q}}\right)$ is a zero of all $G_{11}, \ldots, G_{1 s}$, because $(1, \ldots, 1)$ is a point of $V_{1}$. It is also a zero of all $G_{21}, \ldots, G_{2 t}$, because $\left(\xi^{\gamma_{1}}, \ldots, \xi^{\gamma_{q}}\right)$ is a point of $V_{2}$. Moreover $F_{r}\left(1, \ldots, 1 ; \xi^{\gamma_{1}}, \ldots, \xi^{\gamma_{q}}\right)=1-\xi^{d}=0$. Thus $\left(1, \ldots, 1 ; \xi^{\gamma_{1}}, \ldots, \xi^{\gamma_{q}}\right) \in$ $V$. But $\mathbf{a}^{\prime} \in \mathbb{Z} T_{1} \cap \mathscr{T}_{2}$ means that $\mathbf{a}^{\prime}=\sum_{i=1}^{g} \nu_{i} \mathbf{b}_{1}=\sum_{i=1}^{q} \mu_{j} \mathbf{c}_{\mathbf{j}}$. for suitable integer coefficients $\nu_{i}, \mu_{j}$. Let $\nu=\left(\nu_{1}, \ldots, \nu_{g}\right)$ and $\mu=$ $\left(\mu_{1}, \ldots, \mu_{q}\right)$. Then the second equality shows that the binomial $G=$ $z^{\nu} w^{\mu}-z^{\nu} w^{\mu} \in I(V)$. Moreover $d^{\prime}-\sum_{j=1}^{q} \mu_{j} \gamma_{j}$. But then from $G\left(1, \ldots, 1 ; \xi^{\gamma_{1}}, \ldots, \xi^{\gamma_{a}}\right)=0$, we have that $0=1-\xi^{d^{\prime}}=1-\omega \neq 0$, which is a contradiction.

Corollary 1 . Let $V$ be a simplicial projective toric variety of codimension $r$ over a field of characteristic zero. Then bar $(I(V))=r$ if and only if $V$ is a complete intersection. Moreover, if $F_{1}, \ldots, F_{r}$ are binomials such that $I(V)=\operatorname{rad}\left(F_{1}, \ldots, F_{r}\right)$, then $I(V)=\left(F_{1}, \ldots, F_{r}\right)$ and after a suitable re-arrangement of the indices we have that the binomial 
$F_{i}$ is monic in $y_{i}$ and the other monomial term of $F_{i}$ only involves the $x$-variables and the variables $y_{j}$ such that $j<i$.

Proof. The first part follows from Theorem 1. For the last part, note that in the projective case any two vectors $\mathbf{a}_{\mathbf{1}}, \mathbf{a}_{\mathbf{j}}$ in the parametrization of $V$ can not be proportional. That means that there is no binomial with support $\left\{y_{i}, y_{j}\right\}$. Therefore each binomial $F_{1}, \ldots, F_{r}$ should be monic in $y_{i}$ for some $i$. The proof follows from a careful reading of Lemma 2.

Remark 6. In fact the above Corollary is true for every simplicial toric variety for which each two vectors in $T$ are linearly independent.

\section{POSITIVE CHARACTERISTIC CASE}

Theorem 2. Let $V$ be a simplicial toric variety of codimension $r$ over a field of characteristic $p$. Then bar $(I(V))=r$ if and only if $V$ is completely $p$-glued and $T$ is the p-gluing of $T_{1}$ and $T_{2}$, where $\operatorname{dim}\left(\mathbb{Z} T_{1}\right)=1$ and $\operatorname{dim}\left(\mathbb{Z} T_{2}\right)=n=i m(\mathbb{Z T})$.

Proof. Suppose that $\operatorname{bar}(I(V))=r$. We proceed by induction on $r \geq 1$. The statement is obvious for $r=1$, since in this case $V$ is always a complete intersection and therefore it is also completely $p$-glued, see [9]. Suppose that every simplicial toric variety of codimension smaller than $r$ whose binomial arithmetical rank is equal to the codimension is completely $p$-glued.

We perform the costruction contained in the proof of Lemma 2 for $F_{1}, \ldots, F_{r}$. We suppose that these binomials are arranged in such a way that the corresponding equence of binomial pairs is distinguished with respect to the chain $\Im_{1} \triangleleft \Im_{2} \triangleleft \cdots \triangleleft \Im_{r+1}$.

46rom Lemma 4, Remark 4 and the fact that the Krull dimension of the resi due ring of $I_{T_{1}}$ is equal to $\operatorname{dim}\left(\mathscr{Z T} T_{1}\right)=1$, see [11], we have $\operatorname{bar}\left(I_{T_{1}}\right)=s=(s+1)-1$, therefore $I_{T_{1}}$ is completely $p$-glued by the induction hypothesis. Similarly $\operatorname{bar}\left(I_{T_{2}}\right)=t=(n+t)-n$, therefore $I_{T_{2}}$ is completely $p$-glued by the induction hypothesis.

We complete the proof by semigroup p-gluing. First note that $F_{r}=$ $M_{r}-N_{r} \in I(V)$ and $M_{r}$ only involves variables from $T_{1}$ and $N_{r}$ only variables from $T_{2}$, whence $M_{r}\left(\mathbf{u}^{\mathbf{b}_{1}}, \ldots, \mathbf{u}^{\mathbf{b}_{\mathbf{p}}}\right)=N_{r}\left(\mathbf{u}^{\mathbf{c}_{1}}, \ldots, \mathbf{u}^{\mathbf{c}_{\mathbf{q}}}\right)=\mathbf{u}^{\mathbf{a}}$ for some a $\in \mathbb{N} T_{1} \cap \mathbb{N} T_{2}$. Also there is an element $\mathbf{a}^{\prime} \in \mathbb{Z}^{n}$ such that $\mathscr{Z} \mathbf{a}^{\prime}=\mathscr{Z} T_{1} \cap \mathscr{Z} T_{2}$, since $\mathscr{Z} T_{1} \cap \mathbb{Z} T_{2}$ has dimension 1 .

We claim that there is an integer $k$ such that $\mathbf{a}=p^{k} \mathbf{a}^{\prime}$, i.e. $T$ is the $p$-gluing of $T_{1}$ and $T_{2}$. 
Suppose that our claim is not true, then there exist a positive integer $m>1$ and an integer $k$ such that $\mathbf{a}=m p^{k} \mathbf{a}^{\prime}$ and $p$ does not divide $m$. Note that all entries of $\mathbf{a}$ are positive, so that we can assume that the same is true for $\mathbf{a}^{\prime}$.

Comparing the degrees $d$ of $\mathbf{a}$ and $d^{\prime}$ of $\mathbf{a}^{\prime}$ we have that $d=m p^{k} d^{\prime}$. The number $m$ is not divided by $p$, the characteristic of the field, so let $\omega \in \bar{K}$ be a primitive $m$-th root of unity and let $\xi \in \bar{K}$ be such that $\xi^{d^{\prime}}=\omega$. Re-arrange the coordinates of $\bar{K}^{n+r}$ in order to form the sequence $\left(z_{1}, \ldots, z_{g} ; w_{1} \ldots, w_{q}\right)$. For all $j=1, \ldots, q$, let $\gamma_{j}=\sum_{k-1}^{n} c_{j, k}$. The point $\left(1, \ldots, 1 ; \xi^{\gamma_{1}}, \ldots, \xi^{\gamma_{q}}\right)$ is a zero of all $G_{11}, \ldots, G_{1 s}$, because $(1, \ldots, 1)$ is a point of $V_{1}$. It is also a zero of all $G_{21}, \ldots, G_{2 t}$, because $\left(\xi^{\gamma_{1}}, \ldots, \xi^{\gamma_{q}}\right)$ is a point of $V_{2}$. Moreover $F_{r}\left(1, \ldots, 1 ; \xi^{\gamma_{1}}, \ldots, \xi^{\gamma_{q}}\right)=$ $1-\xi^{d}=0$. Thus $\left(1, \ldots, 1 ; \xi^{\gamma_{1}}, \ldots, \xi^{\gamma_{q}}\right) \in V$. ut $\mathbf{a}^{\prime} \in \mathbb{Z} T_{1} \cap \mathscr{Z} T_{2}$ means that $\mathbf{a}^{\prime}=\sum_{i-1}^{g} \nu_{i} \mathbf{b}_{1}=\sum_{i-1}^{q} \mu_{i j} \mathbf{c}_{j}$, for suitable integer coefficients $\nu_{i}, \mu_{j}$. Let $\nu=\left(\nu_{1}, \ldots, \nu_{g}\right)$ and $\mu=\left(\mu_{1}, \ldots, \mu_{q}\right)$ then the second equality shows that the binomial $G-z^{\nu} w^{\mu}-z^{\nu} w^{\mu} \in I(V)$. Moreover $d^{\prime}=\sum_{j-1}^{q} \mu_{i j} \gamma_{j}$. But then from $G\left(1, \ldots, 1 ; \xi^{\gamma_{1}}, \ldots, \xi^{\gamma_{q}}\right)=0$, we have that $0=1-\xi^{d^{\prime}}=1-\omega \neq 0$, which is a contradiction.

For the converse, let $V$ be a simplicial toric variety of codimension $r$ over a field of characteristic $p$. Let $T$ be the semigroup defining $V$ and suppose that it is completely $p$-glued, and it is the $p$-gluing of $T_{1}$ and $T_{2}$, where $\operatorname{dim}\left(\mathscr{Z} T_{1}\right)=1$ and $\operatorname{dim}\left(\mathbb{Z} T_{2}\right)=\operatorname{dim}(\mathscr{Z T})$. We shall prove that $\operatorname{bar}(I(V))=r$ by induction on $r$

For $r=1$ the claim is obvious, since $I(V)$ is a principal ideal. Therefore $\operatorname{bar}(I(V))=1$. Suppose that the claim is true for all toric varieties of

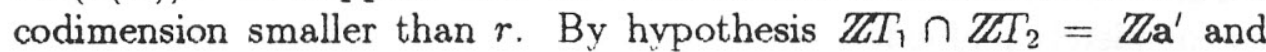

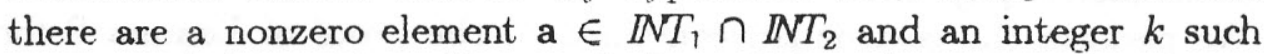
thata $=p^{k} \mathbf{a}^{\prime}$. Let $T_{1}=\left\{\mathbf{b}_{1}, \ldots, \mathbf{b}_{\mathrm{g}}\right\}$, and $T_{2}=\left\{\mathbf{c}_{1}, \ldots, \mathbf{c}_{\mathbf{q}}\right\}$. We call $y_{i}$, for $i=1, \ldots, g$, the variables corresponding to the vectors in $T_{1}$ and $z_{j}$, for $i=1, \ldots, q$, the variables corresponding to the vectors in $T_{2}$. Clearly $I_{T_{1}}=I_{T} \cap K\left[y_{1}, \ldots, y_{g}\right]$ and $I_{T_{2}}=I_{T} \cap K\left[z_{1}, \ldots, z_{q}\right]$. Since a $\in \mathbb{N} T_{1} \cap \mathbb{N} T_{2}$, we can write

$$
\mathrm{a}=\sum \varepsilon_{i} \mathrm{~b}_{1}=\sum \eta_{j} \mathrm{c}_{j}
$$

for some natural numbers $\varepsilon_{i}, \eta_{j}$. This implies that the binomial $F=$ $y_{1}^{\varepsilon_{1}} \ldots y_{g}^{\varepsilon_{g}}-z_{1}^{\eta_{1}} \ldots z_{q}^{\eta_{q}}$ belongs to $I_{T}$. We claim that

$$
I_{T}=\operatorname{rad}\left(I_{T_{1}}+I_{T_{2}}+(F)\right) .
$$


Let $\left(y_{1}, \ldots, y_{g}, z_{1}, \ldots, z_{q}\right) \in \bar{K}^{g+q}$ a zero of $I_{T_{1}}+I_{T_{2}}+(F)$. By hypothesis $\left(y_{1}, \ldots, y_{g}\right)$ is a zero of $I_{T_{1}}$, hence there exist $u_{1}, \ldots, u_{n} \in \bar{K}$ such that $y_{i}=\mathrm{u}^{\mathrm{b}_{\mathrm{i}}}$. For a similar reason there exist $v_{1}, \ldots, v_{n} \in \bar{K}$, such that $z_{j}=\mathbf{v}^{c_{j}}$ for any $i, j$. It follows that $F\left(y_{1}, \ldots, y_{g}, z_{1}, \ldots, z_{q}\right)=$ $0=\mathbf{u}^{\sum \varepsilon_{i} \mathbf{b}_{\mathbf{i}}}-\mathbf{v}^{\sum \eta_{j} \mathbf{c}_{\mathbf{j}}}$. 'T'he ideal $I_{T}$ is generated by binomials of the form:

$$
B=\mathbf{y}^{\gamma_{+}} \mathbf{z}^{\delta_{-}}-\mathbf{y}^{\gamma_{-}} \mathbf{z}^{\delta_{+}} .
$$

We have that $B \in I_{T}$, therefore $\sum \gamma_{+i} \mathbf{b}_{\mathbf{1}}-\sum \gamma_{-i} \mathbf{b}_{\mathbf{i}}=\sum \delta_{+j} \mathbf{c}_{\mathbf{j}}-$ $\sum \delta_{-j} \mathbf{c}_{j} \in \mathbb{Z} T_{1} \cap \mathbb{Z} T_{2}=\mathbb{Z} \mathbf{a}^{\prime}$. Thus there exists a natural number $\tau$ such that $\sum p^{k} \gamma_{1 i} \mathbf{b}_{1}-\sum p^{k} \gamma_{i} b_{1}=\sum p^{k} \delta_{1 j} c_{j}-\sum p^{k} \delta{ }_{j} \mathbf{c}_{j}=$ $p^{k} \tau 20 \mathbf{a}^{\prime}=\tau \mathbf{a}$, in particular we get the relations :

$$
\begin{aligned}
& \sum p^{k} \gamma_{+i} b_{\mathbf{i}}=\sum p^{k} \gamma_{-i} b_{\mathbf{i}}+\sum \tau \varepsilon_{i} b_{\mathbf{i}} \\
& \sum p^{k} \delta_{+j} c_{\mathbf{j}}-\sum p^{k} \delta_{-j} c_{\mathbf{j}}+\sum \tau \eta_{j} \mathbf{c}_{\mathbf{j}}
\end{aligned}
$$

so that

$$
B^{p^{k}}\left(y_{1}, \ldots, y_{g}, z_{1}, \ldots, z_{q}\right)=\mathbf{u}^{\sum p^{k} \gamma_{-i} \mathbf{b}_{\mathbf{1}}} \mathbf{v}^{\sum p^{k} \delta_{-j} \mathbf{c}_{\mathbf{j}}}\left(\mathbf{u}^{\sum \tau \varepsilon_{i} \mathbf{b}_{\mathbf{1}}}-\mathbf{v}^{\sum \tau \eta_{j} \mathbf{c}_{\mathfrak{j}}}\right)=0
$$

Therefore $B \in \operatorname{rad}\left(I_{T_{1}}+I_{T_{2}}+(F)\right)$. The claim follows by the inductive hypothesis since $V\left(I_{T_{1}}\right)$ and $V\left(I_{T_{2}}\right)$ are completely p-glued and whose codimension is smaller than $r$.

\section{EXAMPLES}

In this section we are going to present several examples to clarify the concept of $p$-gluing.

Example 1. There are examples of toric varieties that are completely $p$-glued for every prime number $p$. In [1] we have proved that over a field of positive characteristic all simplicial toric varieties with full support are set-theoretic complete intersections on binomials. It follows from Theorem 2 that every simplicial toric variety with full support is completely $p$-glued, for every $p$. In fact the same proof of Theorem 1 in [1] gives a stronger result:

Let $V$ be a simplicial toric variety of codimension $r$ over a field of characteristic $p$, such that after a suitable re-arrangement of the indices it holds that $\operatorname{supp}\left(\mathbf{a}_{1}\right) \subset \operatorname{supp}\left(\mathbf{a}_{2}\right) \ldots \subset \operatorname{supp}\left(\mathbf{a}_{r}\right)$. Then $V$ is completely 
$p$-glued for every prime $p$ and therefore it is set-theoretic complete intersection on binomials.

Example 2. There are examples of toric varieties that are completely $p$-glued for only one value of $p$. For example, let $V$ be the Veronese surface in $\mathrm{P}^{5}$ parametrized by $x_{1}=t_{1}^{2}, x_{2}=t_{2}^{2}, x_{3}=t_{3}^{2}, y_{1}=t_{2} t_{3}, y_{2}=$ $t_{1} t_{3}, y_{3}=t_{1} t_{2}$. Then $T=\{(2,0,0),(0,2,0),(0,0,2),(0,1,1),(1,0,1)$, $(1,1,0)\}$. According to Theorem 2 , if $V$ were completely $p$-glued for some $p$, then it would be the $p$-gluing of $T_{1}$ and $T_{2}$, where $\operatorname{dim}\left(\mathbb{Z} T_{1}\right)=1$ and $\operatorname{dim}\left(\mathbb{Z T}_{2}\right)=3=\operatorname{dim}(\mathbb{Z} T)$. Each two vectors in $T$ are linearly independent. Therefore $T_{1}$ has just one element. From the definition of $p$-gluing we conclude $t$ hat this element cannot be any of $(2,0,0),(0,2,0),(0,0,2)$, since otherwise $\mathbb{I N T} \cap \mathbb{I N T} T_{2}=\emptyset$. By symmetry, we may assume that $T_{1}=\{(0,1,1)\}$. Easy computations show $\mathrm{t}$ hat $\mathbb{Z} T_{1} \cap \mathbb{Z} T_{2}=\mathbb{Z}(0,1,1)$ and $\mathbb{N} T_{1} \cap \mathbb{I N T} T_{2}={ }^{-} \mathbb{N}(0,2,2)$. Note that $\mathbb{N} T_{2}$ is a complete intersection and therefore completely $p$-glued for every p. But $(0,2,2)=2(0,1,1)$ therefore we conclude that $V$ is completely $p$-glued only for $p=2$.

Example 3. Finally there are examples of toric varieties that are not completely $p$-glued for any $p>0$. For example, let $V$ be the toric variety parametrized by $x_{1}=t_{1}^{2}, x_{2}=t_{2}^{3}, x_{3}=t_{3}^{5}, y_{1}=t_{2} t_{3}, y_{2}=t_{1} t_{3}, y_{3}=$ $t_{1} t_{2}$. Then $T=\{(2,0,0),(0,3,0),(0,0,5),(0,1,1),(1,0,1),(1,1,0)\}$. As in Example 2 one can argue in the following way: if $V$ were $p$-glued for some prime $p$, then the possible partitions for $T$ would be $T_{11}, T_{21}$ or $T_{12}, T_{22}$ or $T_{13}, T_{23}$, where $T_{11}=\{(0,1,1)\}, T_{12}=\{(1,0,1)\}, T_{13}=$ $\{(1,1,0)\}$ and $T_{21}, T_{22}, T_{23}$ are - in the same order - their complements with respect to $T$. Then we would have that $\mathbb{Z} T_{11} \cap \mathbb{Z} T_{21}=\mathbb{Z}(0,1,1)$

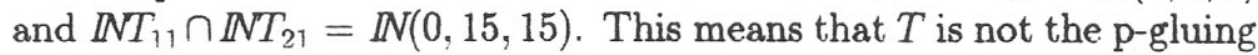
of $T_{11}, T_{21}$. For a similar reason $T$ is not the p-gluing of $T_{12},>T_{22}$ and of $T_{13}, T_{23}$, since $\mathbb{Z} T_{12} \cap \mathbb{Z} T_{22}=\mathbb{Z}(1,0,1), \mathbb{N} T_{12} \cap \mathbb{I N T} T_{22}=\mathbb{N}(10,0,10)$, $\mathscr{Z} T_{13} \cap \mathbb{Z} T_{23}=\mathbb{Z}(1,1,0)$ and $\mathbb{N} T_{13} \cap \mathbb{N} T_{23}=\mathbb{N}(6,6,0)$. None of $15,10,6$ is a power of a prime number, therefore we conclude that $V$ is not completely $p$-glued for any $p$.

\section{REFERENCES}

[1] M. Barile, M. Morales, A. Thoma, On Simplicial Toric varieties which are Set-Theoretic Complete Intersections, Journal of Algebra, (to appear).

[2] E. Becker, R. Grobe, M. Niermann, Radicals of binomial ideals, J. Pure and Applied Algebra 117 \& 118, (1997), 41-79. 
[3] Ch. Delorme, Sous monoides d'intersection complete de $\mathbb{I N}$, Ann. scient. Ec. Norm. Sup., 4 serie, 9, (1976) 145-154.

[4] D. Eisenbud and B. Sturmfels, Binomial ideals, Duke Math. J. 84,(1996) 1-45.

[5] S. Eliahou and R. Villarreal, On systems of binomials in the ideal of a toric variely, preprint.

[6] K. Fischer, W. Morris, J. Shapiro, Affine semigroup rings that are complete intersections, Proc. Amer. Math. Soc. 125(1997) 3137-3145.

[7] T. T. Moh, Set-theoretic complete intersections, Proc. Amer. Math. Soc. 94 (1985), 217-220.

[8] J. C. Rosales, On Presentations of subsemigroups of $\mathbb{N}^{n}$, Semigroup Forum, 55 (1997), 152-159.

[9] J.C. Rosales and P. A. Garcia-Sanchez, On complete intersection affine semigroups, Commun. Algebra, 23(14) (1995) 5395-5412.

[10] G. Scheja, O. Scheja, U. Storch, On regular sequences of binomials, Manuscripta Math. 98 (1999) 115-132.

[11] B. Sturmfels, Gröbner Bases and Convex Polytopes. University Lecture Series, No. 8 American Mathematical Society Providence, R.I. 1995.

[12] A. Thoma, On the binomial arithmetical rank, Arch. Math. 74 (2000) 22-25. 